\title{
Contribution to the Understanding of Desolvation Mechanisms: Impact of Crystal Size, Structural Purity and Process
}

\author{
S. Petit, G. Coquerel \\ Sciences et Méthodes Séparatives, IRCOF, University of Rouen, F76821 Mont Saint Aignan Cedex, France \\ gerard.coquerel@univ-rouen.fr
}

\begin{abstract}
In the literature, the models and classifications devoted to the desolvation mechanisms often consider the incidence of structural features or the knowledge derived from kinetic studies, but have poorly recognised the impact of physical and macrocrystalline factors. Based on experimental studies and presumed mechanisms, it is shown here that crystal size, punctual or lattice defects, and structural purity may have a dramatic influence on the desolvation behaviour of organic and pharmaceutical crystals. As a consequence, the preparation route of each sample and the eventual physical treatments should be explicitly mentioned and taken into account for a satisfactory interpretation of experimental data.
\end{abstract}

Keywords:

Desolvation Process, Physical treatment, Particle size, Crystal defects, Process-induced transformation

\section{INTRODUCTION}

The crystallisation of organic compounds in solution can lead to different crystalline phases, depending on the experimental conditions: temperature, supersaturation, kinetics, nature of the solvent, presence of additives and/or impurities, etc. [1]. These distinct crystalline forms usually exhibit different physico-chemical properties (solubility, stability, melting point, etc.), which may have a dramatic incidence on their behaviour for specific applications, for instance in the pharmaceutical domain (dissolution rate, bioavailability, evolution upon storage, etc.) [2].

If all the isolated crystalline forms of a solute crystallise without incorporation of solvent molecules in their crystal lattice, this so-called polymorphic behaviour can be further investigated by a study of stability relationships (monotropy or enantiotropy) between these phases [3], so as to control the eventual spontaneous transition from a less stable toward a more stable polymorph.

In the case of hydrates and solvates, the questions related to the relative stability of different crystalline phases are more complex and most often, only hydrates or non solvated forms solids can be considered as suitable forms for the pharmaceutical development [4]. As a consequence of the necessity to perform a systematic research of polymorphic forms and solvates during early stages of pharmaceutical development $[5,6]$, the number of solid phases identified as hydrates and solvates has increased continuously during the last twenty years, which can be detected from the proportion of solvates among the crystal structures deposited in the Cambridge Structural Database (CSD) [7]. This increased proportion of solvates and the related structural knowledge revealed the diversity of the roles played by solvent molecules in crystal packings [8], and also provides new opportunities to investigate the mechanism of solid-solid transitions occurring during the desolvation of these crystalline phases. When solvates have to be considered during the development of pharmaceutical ingredients, two situations are usually encountered:
- the solvent molecules need to be removed from the solid in order to access to an acceptable pure component.

- the final product is itself a solvate (often a hydrate) and unwillingly the solid continuously adapts to environmental conditions, leading to a shift in physical properties.

The present paper intends to propose an extension of previous studies on this topic by considering the incidence of a few physical parameters on the presumed desolvation mechanisms.

\section{LITERATURE SURVEY AND BACKGROUND}

During the $20^{\text {th }}$ Century, the fundamental aspects of polymorphic transitions have been analysed by many authors, giving rise to several models and theoretical interpretations [9]. Although several distinct theories have been proposed, the current knowledge in this field has been considered sufficient to envisage an explanation of polymorphic transitions induced by industrial treatments and processes [10]. In the examples given by Morris et al., it can be highlighted that humidity or solvent vapour pressure can play an important role, together with other parameters (temperature, kinetics, ...) that are involved in industrial processes such as grinding, milling, spray-drying, freeze-drying, etc. However, this theoretical approach of process-induced polymorphic transitions has not been extended to desolvations, probably because of the significant differences between the related mechanisms.

In 1982, S. Byrn was probably one of the first to propose a global approach of the dehydration of organic crystals [11]. Considering rather simple compounds such as caffeine, theophylline, thymine or cytosine, he used a combination of structural data and experimental observations (mainly optical observations) to elaborate a rational description of macroscopic consequences of dehydrations on basis of structural features.

In 1996, we have proposed a unified model (so-called hereafter Rouen-96) for the dehydration of molecular

This is an Open Access article distributed under the terms of the Creative Commons Attribution-Noncommercial License (http://creativecommons.org/licenses/by-nc/3.0/), which permits unrestricted use, distribution, and reproduction in any noncommercial medium, provided the original work is properly cited. 
crystals [12]. Assuming that a dehydration process can be divided into two steps corresponding to the successive release of water molecules and to a possible reorganisation step, it was suggested that two structural criteria could be used to distinguish destructive and cooperative processes for the departure of water molecules. In case of non destructive departure, the possibility of a cooperative reorganisation step can then be assessed by considering two supplementary criteria related to the physical features (cristallinity and size of domains) of the anhydrous material. In case of destructive release of water, or of non cooperative reorganisation, the only pathway for the evolution toward a crystalline desolvated phase is a nucleation and growth process, and the absence of reorganisation should induce that an amorphous material is obtained. Whatever the type of reorganisation, the destructive character of the global process is characterized by the absence of structural filiation between the mother and daughter phases, whereas a cooperative process of release of solvent and reorganisation (reached if the four successive criteria are fulfilled) induces the transmission of structural information along the dehydration mechanism.

K. Morris, in 1999, has proposed a structural classification of hydrates [13], in which one can retrieve some of the basic notions used in the Rouen-96 model. In this approach, the environment of water molecules in crystals structures is correlated to the thermal behaviour of the corresponding phases, with the assumption that hydrates can be classified in three groups, namely 'isolated site hydrates' (class 1) in which water molecules are encapsulated by neighbouring molecules, 'channel hydrates' (class 2) where an easy and therefore cooperative release of water molecules can occur, and 'ion associated hydrates' (class 3) for which the existence of strong bonds with cations induces a high dehydration temperature.

Using mainly data derived from kinetic studies, A. Galwey has published in 2000 a detailed model of dehydrations based on the concept of 'Water Evolution Type' [13]. From a macroscopic analysis of dehydrations often based on a combination of thermal behaviour, kinetic measurements and microscopic observations, Galwey has described a series of phenomena ranging from a diffusive departure of water molecules, sometimes with a topotactic character, to the displacement of a reaction interface producing an amorphous material or associated to a recrystallisation at this interface. It is noteworthy that despite the absence of a structural approach in the model of Galwey, some common interpretations are in both models Rouen-96 and Galwey.

In all the models elaborated so far, as well as in descriptive classifications based for instance on the strength of the interactions between solvent molecules and 'host' crystalline networks [15], it is more or less explicitly assumed that a given solvated phase should undergo a single desolvation behaviour, whatever the preparation route, the physical characteristics of the used sample, and the experimental conditions associated to the desolvation. This important point can be exemplified by the case of trehalose dihydrate, for which it was demonstrated that kinetic conditions could induce significant changes in the dehydration behaviour, leading either to an amorphous solid or to the anhydrous phase $\alpha$ [16]

The necessity to define and to take into account the history of each sample, its physical features and the incidence of experimental conditions of desolvation is, to our opinion, a major weakness of the existing models. However, we should also be aware of other limitations that affect the structural models. For instance, in the model Rouen-96, the arbitrary separation between departure of water molecules and reorganisation step may constitute a difficulty in cases where these two steps occur simultaneously and possibly in an interdependent way. It should also be kept in mind that structural approaches of desolvations implicitly envisage the initial phase as a perfect crystal without defects nor impurities and without surfaces ..

\section{EXAMPLES AND DISCUSSION}

\subsection{Understanding a weight loss in an industrial process}

In a crystallization process performed by cooling in ethyl acetate from $45^{\circ} \mathrm{C}$ to $0^{\circ} \mathrm{C}$ on a particular weak organic acid and an organic base, it appeared that a large weight loss (up to $12 \% \mathrm{w} / \mathrm{w}$, specification $<1.5 \%$ ) occurred upon drying at reduced pressure, due to a partial release of the base. Interestingly, this problem could be solved by adding a supplementary step in the process, consisting of a vigorous milling of the suspension by using ultra-turrax, followed by a period of at least twelve hours at low temperature (between $0^{\circ} \mathrm{C}$ and $10^{\circ} \mathrm{C}$ ) prior to filtration.

Further investigations were carried out in order to account for the beneficial effect of the milling step, including careful optical microscopy observations of solid samples before and after the treatment. From this analysis, it could be established that the vigorous reduction of the mean particle size $(<30 \mu \mathrm{m})$ and the formation of high energy surface induced a 're-growth' phenomenon of ground particles in smoother conditions of supersaturation, leading to larger crystalline particles with an improved morphology and structural quality, a higher uniformity and a lower proportion of defects.

This example illustrates that active pharmaceutical ingredients can be strongly sensitive to manufacturing conditions, including drying, grinding and storing. A theoretical and general approach of the consequences of manufacturing conditions in terms of polymorphic behaviour was proposed a few years ago by Morris and coworkers [10], but, to our knowledge, the specific case of solvates, including the incidence of physical factors on the desolvation mechanism, was not yet envisaged.

\subsection{Incidence of crystal size}

In the course of experimental investigations, it is sometimes difficult to correlate the observations performed with different techniques, for instance DSC (that requires a finely ground powder) and hot stage optical microscopy (for which one or a few large single particles are more suitable). Such discrepancies may be due to the incidence of the particle size distribution of each sample on the desolvation process, even in cases where the mechanism involved at the molecular scale is not modified. This can be easily understood by considering a desolvation process involving, during its first stage, the formation of a thin (but macroscopic) waterproof - or solvent-proof - layer at the surface of each particle.

As shown in Figure 1, the desolvated layer may prevent further release of solvent and may induce, at higher temperature, a side effect consisting of the partial dissolution of the solute, associated to an increase of pressure in the inner part of the particle. When both temperature and/or internal pressure reach a critical value, a sudden endothermic phenomenon may be observed, corresponding to the formation of macroscopic cracks allowing the evacuation of the vaporised solvent. 


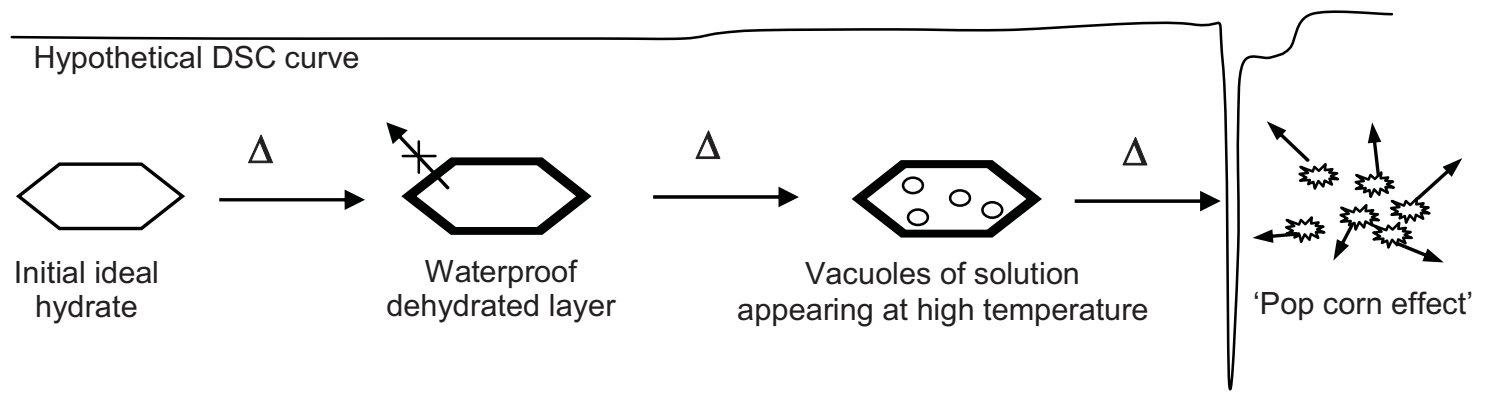

Figure 1: Schematic illustration of an unusual dehydration mechanism

in case of formation of a waterproof layer at the surface of large particles

This explosive disruption of the particles is colloquially labelled 'pop corn effect', and may also, in some cases, be associated to a chemical degradation caused by the too fast heating that prevents a progressive and smooth release of solvent molecules.

This type of situation has been observed during the thermal decomposition of the DMSO solvate of cortisone acetate [17]. It was highlighted in the associated discussion that structural data did not allow the prediction of this series of events, and that structural models are unable to account for phenomena directly related to the crystal size distribution. It should also be mentioned that, in case of formation of a solvent-proof layer, the desolvation mechanism is determined by the particle size and quality, since large particles with a higher proportion of defects are more likely to produce more complex behaviours, whereas there is no formation of solvent-proof layer if the size of the particles is smaller than twice the width of the layer.

\subsection{Incidence of crystal defects}

In molecular crystals, macroscopic defects can be sometimes carefully characterised [18], but the nature and the proportion of microscopic defects is more difficult to elucidate. However, it has been long ago recognised that both crystallisation conditions and additional physical, thermal or mechanical treatments may have an impact in terms of nature and number of defects $[19,20]$, which could in turn induce significant changes of thermal behaviours, physical properties and stability, as illustrated by the situation depicted in section 3.1. Regarding the desolvation phenomena, a homogeneous distribution of defects may be responsible for a shift in the desolvation peak detected in DSC, referred to an identical non defective sample (Figure 2).

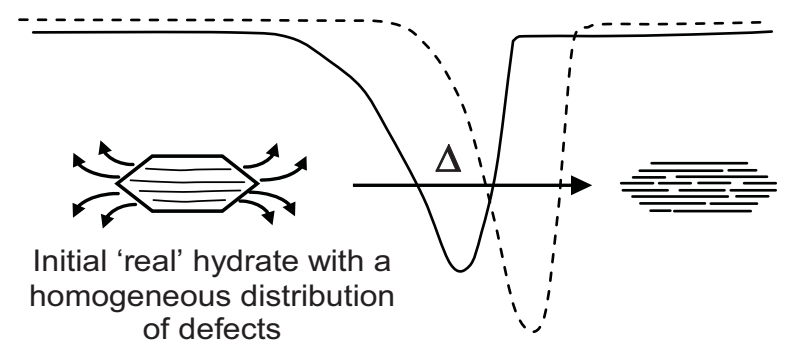

Figure 2: Incidence of a large proportion of crystal defects on DSC curves (the broken line represents the case of an ideal crystal without defect).

Such behaviour can be exemplified with a-lactose monohydrate, since our recent study has shown that manual grinding induces a decrease of about $20^{\circ} \mathrm{C}$ of the dehydration temperature [21]. Interestingly, it was observed in this work that grinding does not produce a continuous shift toward lower temperatures of the dehydration phenomena observed in DSC, but a progressive replacement of the initial thermal event by a distinct event. This was interpreted as a change of the dehydration mechanism caused by grinding, and further characterisation of native and ground samples by high-resolution X-ray diffraction, NMR, SEM and determination of PSD were used to investigate the nature of crystal defects, in particular in terms of crystal lattice distortions [22].

\subsection{Incidence of structural purity}

Assuming that its chemical purity is not questionable, a sample is said of high structural purity if it presents the same 3D molecular arrangement in every particle [23]. In the frame of this definition, the two factors envisaged in the previous sections have no direct relationship with structural purity. Indeed, the crystal size distribution does not impact on the structural purity, and the existence of disorder or crystal defects should be clearly differentiated from the structural purity. Actually, a defective or poorly crystalline sample is supposed to be structurally pure if the spatial distribution of disorder or defects is homogeneous within the sample. By contrast, a sample formed by the physical mixture of a well-crystallized solid and an amorphous material exhibits a poor structural purity.

Hence, the concept of structural purity relies on the homogeneous distribution of structural features, including mixtures of phases, partial amorphisations, heterogeneous distributions of defects, etc. In the context of desolvation behaviours, the existence of heterogeneities in the solvated material may lead to complex and hardly interpretable thermal behaviours, as exemplified in Figure 3. Beside usual factors that may affect the structural purity, specific heterogeneities can be encountered in solvates, such as displacements of solvent molecules from a crystallographic site to another, depending mainly on the vapour pressure of the solvent, and on its affinity with water. These parameters may be responsible, in extreme cases, for progressive solvent exchanges [24].

\section{CONCLUSION}

The study of desolvation phenomena is usually performed on the basis of structural data, or through the elucidation of stability relationships between the solid phases, together with their characterisation. These complementary approaches constitute two fundamental methodological supports for the understanding of desolvations, and can be founded on structural information or on phase diagrams. However, a third set of data should be considered for a more satisfactory analysis of desolvation behaviours, consisting of the physical factors that may impact on the mechanism of solid-solid transformations: crystal size and structural purity nature. 


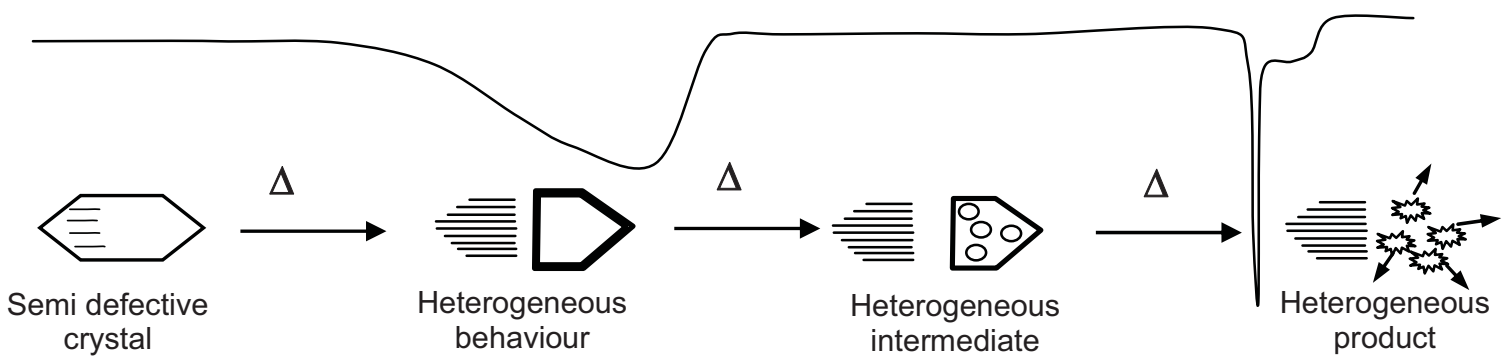

Figure 3: Schematic illustration of an unusual dehydration mechanism: hypothetical case of a heterogeneous behaviour caused by an irregular distribution of crystal defects.

Although the structural approach has proven its contribution to the rationalisation of desolvation phenomena, it must also be kept in mind that crystal structures deduced from diffraction studies correspond to an idealised representation of the physical state of a sample that necessarily contains a large proportion of heterogeneities. When imperfections of crystalline particles can interfere in a desolvation process, the rationalisation of the underlying mechanism can only be reached if the physical factors of the 'third set of data' are explicitly considered during the interpretation of experimental data.

\section{REFERENCES}

[1] Boistelle R., Astier, J.P., 1988, Crystallization mechanisms in solution. J. Crystal Growth, 90, 14-30.

[2] Hilfiker R., Blatter F., von Raumer M., 2006, Relevance of solid-state properties for pharmaceutical products (chap.1), in Polymorphism in the Pharmaceutical Industry, R. Hilfiker (ed.), Wiley-VCH, Weinheim, pp. 1-19.

[3] Burger A., Ramberger R., 1979, On the polymorphism of pharmaceuticals and other molecular crystals, Mikrochim. Acta, 2, 259-271 \& 273-316.

[4] Griesser U., 2006, The importance of solvates (chap.8), in Polymorphism in the Pharmaceutical Industry, R. Hilfiker (ed.), Wiley-VCH, Weinheim, pp. 211-233.

[5] Giron D., Mutz M., Garnier S., 2004, Solid state of pharmaceutical compounds. Impact of $\mathrm{ICH}$ Q6 guidelines on industrial development, J. Therm. Anal. Cal., 77, 709-747.

[6] Byrn S.R., Pfeiffer R.R., Ganey M., Hoiberg C., Poochikian G., 1995, Pharmaceutical solids: a strategic approach to regulatory considerations, Pharm. Res., 12, 945-954.

[7] Görbitz C.H., Hersleth H.P., 2000, On the inclusion of solvent molecules in the crystal structures of organic compounds, Acta Crystallogr., B56, 526-534.

[8] Van der Sluis P., Kroon J., 1989, Solvents and X-ray crystallography, J. Cryst. Growth, 97, 645-656.

[9] Mnyukh Y.V., 1979 Molecular mechanism of polymorphic transitions, Mol. Cryst. Liq. Cryst., 52, 163-200.

[10] Morris K.R., Griesser U.J., Eckhardt C.J., Stowell J.G., 2001, Theoretical approaches to physical transformations of active pharmaceutical ingredients during manufacturing processes, Adv. Drug Deliv. Rev., 48, 91-114.

[11] Byrn, S. R., 1982, Loss of Solvent of Crystallization (chap.5), in Solid-State Chemistry of Drugs, Academic Press, New York, pp.149-188.
[12] Petit S., Coquerel G., 1996, Mechanism of several solid-solid transformations between dihydrated and anhydrous copper(II) 8-hydroxyquinolinates. Proposition for a unifiedmodel for the dehydration of molecular crystals. Chem. Mater., 8, 2247-2258.

[13] Morris, K.R., 1999, Structural aspects of hydrates and solvates, in Polymorphism in Pharmaceutical Solids, 95, Brittain H.G. (Ed.), Marcel Dekker, New York, pp. 125-181.

[14] Galwey A.K., 2000, Structure and order in thermal dehydrations of crystalline solids, Thermochim. Acta, $355,181-238$

[15] Mimura H., Kitamura S., Kitagawa T., Kohda S., 2002 Characterization of the non-stoichiometric and isomorphic hydration and solvation in FK041 clathrate, Colloids and Surfaces B: Biointerfaces, 26, 397-406.

[16] Willart J.-F., De Gusseme A., Hemon S., Descamps M., Leveiller F., Rameau A., 2002, Vitrification and polymorphism of trehalose induced by dehydration of trehalose dihydrate, J. Phys. Chem., B 106, 33653370

[17] Petit S., Mallet F., Petit M.-N., Coquerel G., 2007, Role of structural and macrocrystalline factors in the desolvation behaviour of cortisone acetate solvates, J. Therm. Anal. Cal., 90, 39-47.

[18] Berton B., Dupray V., Atmani H., Coquerel G., 2007, Gas vacuoles formation during the dehydration of trehalose dihydrate: A Raman microspectroscopy approach, J. Therm. Anal. Cal., 90, 325-328.

[19] Hüttenrauch R., Keiner I., 1979, Produce lattice defects by drying process. Int. J. Pharm., 2, 59-60.

[20] Hüttenrauch R., Fricke S., Zielke P., 1985, Mechanical activation of pharmaceutical systems, Pharm. Res., 302-306.

[21] Garnier S., Petit S., Mallet F., Petit M.-N., Lemarchand D., Coste S., Lefebvre J., Coquerel G., 2008, Influence of ageing, grinding and preheating on the thermal behaviour of a-lactose monohydrate, Int. J. Pharm., 361, 131-140.

[22] Fukuoka E., Terada K., Makita M., Yamamura S., 1995, Paracrystalline lattice distortion in crystalline pharmaceuticals. Determination of paracrystalline lattice distortion by powder X-ray diffraction, Chem. Pharm. Bull., 43, 671-676.

[23] Coquerel G., 2006, The 'structural purity' of molecular solids - an elusive concept?, Chem. Engin. Proc., 45, 857-862.

[24] Mallet F., Petit S. Lafont S., Billot P., Lemarchand D., Coquerel, G., 2003, Solvent exchanges among molecular compounds. Two extreme cases of pharmaceutical interest, J. Therm. Anal. Cal., 73, 459471. 